\title{
Quality of Life and Depression in Rheumatoid Arthritis Patients Treated with Biologics - A Single Centre Experience
}

\author{
Wojciech Tański', Adrianna Szalonka ${ }^{2}$, Beata Tomasiewicz' \\ 'Department of Internal Medicine, 4th Military Teaching Hospital, Wroclaw, Poland; ${ }^{2}$ Centre for Research and Innovation, 4th Military Teaching \\ Hospital, Wroclaw, Poland \\ Correspondence: Wojciech Tański, Department of Internal Medicine, 4th Military Teaching Hospital, Weigla 5, Wroclaw, 50-98I, Poland, \\ Email dr.wojciech.tanski@gmail.com
}

\begin{abstract}
Introduction: Patients with rheumatoid arthritis (RA) often experience depression, which has a very negative impact on the assessment of the quality of life (QoL). However, there are not many studies that assess the relationship between depression and QoL in RA patients. The aim of the study was to assess the level of QoL and determine the mutual relationship between anxiety and depression levels and QoL in patients treated for RA.
\end{abstract}

Material and Methods: The study included 101 patients (aged:52.4 \pm 16.97 ), who met the established criteria for a diagnosis of RA and treatment with a biological agent. Only standardized tools were used to examine the patients: WHO-QoL, HADS and the VAS scale.

Results: The mean RA duration in the group studied was $13.54 \pm 9.51$ years and the disease activity score was $4.8 \pm 0.8$. The mean QoL perception score was $3.48 \pm 0.8$. Nearly $40 \%$ of the respondents could not clearly determine their QoL, perceiving it as neither poor nor good, and $10 \%$ believed their QoL is poor or very poor. The correlation analysis revealed that anxiety is significantly and negatively associated with QoL in the psychological domain $(r=-0.472, p<0.001)$ and social domain $(r=-0.298, p=0.023)$ and depression is significantly and negatively associated with QoL in the psychological domain $(r=-0.322, p=0.01)$ and physical health domain $(r=-0.209, p=0.04)$. In the multiple linear regression model, depression was an independent negative predictor affecting the following domains: perception of QoL and perception of health, and physical health.

Conclusion: RA patients treated with biologics present a low level of health perception and an average level of QoL perception. Depression and anxiety negatively correlate with QoL domains: the higher the anxiety and depression levels, the poorer the QoL in the psychological and social relationships domains. Depression is an independent determinant of decreased QoL.

Keywords: rheumatoid arthritis, quality of life, depression, anxiety, predictors

\section{Background}

The most common form of arthritis is rheumatoid arthritis (RA), which affects 1.5 million adult Americans. ${ }^{1-3}$ In contrast to the widespread stereotypes, RA also develops in young people aged 30-50 years. In Poland, RA affects $0.9 \%$ of the population and it is 3 times more prevalent in women. In a small group of patients (10-20\%), the onset of the disease occurs after the age of 65 , with a reverse female-to-male ratio of prevalence as compared to young patients. The disease causes pain and limits physical performance. Patients with RA experience increased disability and exacerbation of RArelated comorbidities. ${ }^{4}$ Apart from somatic symptoms and physical limitations, the disease is accompanied by chronic fatigue and low mood. ${ }^{5}$ Progressive disability associated with arthritis may lead to negative psychological consequences, from sadness, through depressive symptoms, to depression, including clinical depression. ${ }^{6}$

The subject literature shows that depression occurs in $4.0-66.2 \%$ of RA patients. This is a considerably higher prevalence as compared to the population without $\mathrm{RA}^{7}$ or patients with arthritis of different aetiology. ${ }^{8}$ The published results also show that arthritis has a significant, strong impact on the quality of life $(\mathrm{QoL})$ assessment in arthritis patients 
as compared with the general population. Likewise, people with depression have a lower self-assessed QoL than individuals without depression. It has been demonstrated that compared to other chronic diseases, depression has the greatest negative influence on patient QoL. Therefore, patients with concomitant depression and arthritis may have lower QoL than patients with only one of these diseases. ${ }^{9}$ RA patients experience lower health related quality of life (HRQoL) than that observed in people suffering from other rheumatic conditions or in healthy individuals. Even when RA is well controlled, these patients may still experience reduced HRQoL. ${ }^{10}$

The goal of treating RA is to select such treatment that would allow for controlling the patient's well-being and disease activity, resulting in the best possible remission, low disease activity and high level of quality of life. In the therapeutic process, it is important to monitor the safety of the treatment used and comorbidities. Biological treatment is increasingly used in patients with RA who express great approval for the therapy, despite its side-effects. Apart from the traditional measures of the clinical activity of the disease, patient-reported results and QoL are becoming important parameters in the assessment of treatment response among RA patients. The treatment used may have an effect on the subjective perception of one's health. Therefore, treatment of rheumatic disorders should be administered by an interdisciplinary team lead by a rheumatologist. According to international guidelines, such a team should include a neurologist, a psychiatrist, a psychotherapist, a rehabilitation specialist, a social worker, an orthopaedist, a primary care physician, a nurse, a dietician, and a pharmacist.

In light of the above-listed information and the available publications, which most often assess QoL or depression in RA patients, and given the scarce number of studies assessing the relationship between depression and QoL in RA patients, it seems important to conduct a study, whose aim is to verify how the anxiety and depression levels experienced by this group of patients affect the perception of QoL.

Thus, the aim of the study is to assess the level of QoL and to determine the mutual relationship between anxiety and depression levels and QoL in patients treated for RA.

The following hypotheses were adopted in the study:

1. HRQoL in RA patients is low, and their levels of depression and anxiety are high.

2. There is a significant relationship between anxiety and depression levels and QoL; the higher the anxiety/ depression level, the lower the QoL.

Additionally, the study analysed the intensity of pain and its relationship with the QoL assessment.

\section{Study Material}

\section{Study Group}

Respondents to a screening questionnaire had to meet the following

\section{Eligibility Criteria}

- Age $\geq 18$ years,

- Diagnosis of RA,

- Pharmacological treatment with a biologic in accordance with the guidelines by the American Rheumatism Association,

- Informed voluntarily consent to participate in the study,

- Ability to independently fill out the survey questionnaires.

\section{Exclusion Criteria}

- Age $<18$ years,

- Diagnosed and pharmacologically treated mental disorders (depression or mental diseases involving low mood),

- No possibility of independently filling out the survey questionnaires,

- Coexistence of other chronic illnesses that may disturb the emotional state and QoL (cancer, renal failure, NYHA class IV heart failure). 


\section{Instruments}

WHOQOL-BREF- This generic questionnaire comprises 26 items and measures six following domains: overall perception of QoL, overall perception of one's health, physical health, psychological, social relationships, and environment. The measurement pertains to the past 14 days. The scores of items in each domain are summed up and then converted into a 0-100 scale. The higher the score, the better the HRQoL. The WHOQOL-BREF is an instrument with a good Internal consistency, sensitivity to change, as well as discriminant validity, which means that it has an excellent ability to discriminate between subjects who are ill and those who are well. ${ }^{11}$

Hospital Anxiety and Depression Scale (HADS) - This instrument comprises 14 items and is used to measure anxiety and depression. ${ }^{12}$ The respondents answer questions about depression and anxiety in the preceding week, which are scored from 0 to 3. Items in the subscales are summed, with the score range of 0-21. The higher the score, the worse the mood. Scores in the range of 0-7 are regarded as "normal", 8-10 as "borderline", and >11 as "abnormal" ("caseness"). The instrument is a well-established tool, presenting high test-retest reliability and strong internal consistency. ${ }^{12}$

Visual Analogue Scale - VAS - This is a reliable tool used to assess the intensity of pain. The cyclically repeated measurements of pain intensity with the scale allow for assessing the effectiveness of the analgesic treatment used. The scale has a form of a 10-cm ruler, with 0 denoting no pain and 10 the worst pain imaginable. As the tool is very clear to most patients, and presents considerable reliability and measurement repetitiveness, it is the most commonly used scale in pain assessment. ${ }^{13}$

\section{Study Protocol}

The study was conducted in a centre specialising in biological treatment. The centre has extensive experience in biological drug programs, which it has implemented for many years. As part of the centre's activity, patients receive treatment with various biological agents. The majority of patients included in the study had been treated with tocilizumab due to the failure of standard medications or TNF-alpha inhibitors. In most of the cases, tocilizumab was used as monotherapy, but concomitant treatment mostly with methotrexate was also used. The data were obtained from an observational cross-sectional study involving consultation of RA patients undertaken from September 2020 to September 2021. Patients invited to participate in the study had been treated in the rheumatology centre offering biological therapy and attended follow-up visits as part of their biological treatment. Not all the patients invited to participate in the study $(\mathrm{n}=126)$ filled out the questionnaires. Thus, the final number of patients included in the analysis was $n=101$. The patients were examined by a team comprising a rheumatologist and an internist nurse, who had been trained on how to perform the tasks within the present study. The physician assessed the patients' clinical data (clinical examination, clinical history, VAS and DAS-28 assessment). The nurse's role was to support the patient if they had any questions regarding the questionnaires and the study. The main objective was to obtain questionnaires that the patients completed themselves. The clinical data came from the patients' medical documentation (disease duration, number of hospitalizations in the preceding 12 months, and the treatment used).

The patients were informed of the purpose and course of the study and of the fact that they could withdraw from the study at any stage. The study was approved by the relevant Bioethics Committee (No. 170/2020) and was conducted in accordance with the Helsinki Declaration and the principles of good clinical practice, with respect for the rights and dignity of the participants.

\section{Description of the Statistical Methods Used}

An analysis of quantitative variables (ie those expressed numerically) was performed by calculating the mean, standard deviation, median, and quartiles. An analysis of qualitative variables (ie those expressed non-numerically) was performed by calculating the number and percentage of occurrences of each of the values. Correlations between the quantitative variables were calculated using the Spearman correlation coefficient. Multiple linear regression analysis was used to assess the impact of several variables on a given quantitative variable. The results were presented as parameter values in a regression model with a confidence interval of $95 \%$. A significance level of 0.05 was adopted in the study. This means that all $\mathrm{p}$ values below 0.05 were interpreted as an indication of significant relationships. The analysis was performed using the R-4.1.1. statistical software. ${ }^{14}$ 
Table I Socio-Clinical Characteristics of the Study Group

\begin{tabular}{|c|c|c|}
\hline \multicolumn{2}{|l|}{ Parameter } & \multirow{4}{*}{$\begin{array}{l}\text { Total } \\
52.38 \pm 16.97 \\
55 \\
39.5-64\end{array}$} \\
\hline Age [years] & Mean $\pm S D$ & \\
\hline & Median & \\
\hline & Quartiles & \\
\hline \multirow[t]{3}{*}{ Disease duration [years] } & Mean \pm SD & $|3.54 \pm 9.5|$ \\
\hline & Median & 10 \\
\hline & Quartiles & $7-19$ \\
\hline \multirow[t]{2}{*}{ Relationship status } & In a relationship & $63.37 \%$ \\
\hline & Single & $36.63 \%$ \\
\hline DAS-28 & Mean $\pm S D$ & $4.8 \pm 0.8$ \\
\hline \multirow[t]{2}{*}{ Gender } & Female & $62.90 \%$ \\
\hline & Male & $37.10 \%$ \\
\hline \multirow[t]{3}{*}{ BMI } & Normal weight & $58.12 \%$ \\
\hline & Overweight & $27.59 \%$ \\
\hline & Obesity & $14.29 \%$ \\
\hline \multirow[t]{9}{*}{ Comorbidities } & Hypertension & $20.79 \%$ \\
\hline & Diabetes & $7.92 \%$ \\
\hline & Atherosclerosis & $4.95 \%$ \\
\hline & Asthma & $5.94 \%$ \\
\hline & Osteoporosis & $0.99 \%$ \\
\hline & Hypothyroidism & $17.82 \%$ \\
\hline & Sjogren's syndrome & $2.97 \%$ \\
\hline & Psoriasis & $20.79 \%$ \\
\hline & None & $38.61 \%$ \\
\hline \multirow[t]{6}{*}{ Biologics treatment } & Certolizumab & $6.93 \%$ \\
\hline & Etanercept & $14.85 \%$ \\
\hline & Adalimumab & $2.97 \%$ \\
\hline & Golimumab & $1.98 \%$ \\
\hline & Baricitinib & $5.94 \%$ \\
\hline & Tocilizumab & $67.33 \%$ \\
\hline VAS & Mean \pm SD & $4.4 \pm 2.7$ \\
\hline \multirow[t]{4}{*}{ HADS: anxiety } & Mean $\pm S D$ & $20.0 \pm 2.3$ \\
\hline & Normal & $65.08 \%$ \\
\hline & Borderline & $28.57 \%$ \\
\hline & Abnormal & $6.35 \%$ \\
\hline \multirow[t]{4}{*}{ HADS: depression } & Mean $\pm S D$ & $17.1 \pm 2.2$ \\
\hline & Normal & $71.42 \%$ \\
\hline & Borderline & $14.29 \%$ \\
\hline & Abnormal & $14.29 \%$ \\
\hline \multirow[t]{6}{*}{ WHOQOL-BREF } & Perception of health & $2.63 \pm 1.0$ \\
\hline & Perception of quality of life & $3.48 \pm 0.8$ \\
\hline & Physical health domain & $11.0 \pm 2.44$ \\
\hline & Psychological domain & $17.0 \pm 2.12$ \\
\hline & Social relationships domain & $15.5 \pm 2.09$ \\
\hline & Environment domain & $14.0 \pm 2.17$ \\
\hline
\end{tabular}

Abbreviations: SD, standard deviation; WHOQOL-BREF, The World Health Organization Quality of Life Questionnaire; VAS, Visual Analogue Scale; HADS, Hospital Anxiety and Depression Scale; DAS 28, Disease Activity Score. 


\section{Characteristics of the Study Group}

The mean age of the study group was $52.4 \pm 16.97$ years, the majority of patients were in a relationship (63.37\%), and had a normal BMI (58.12\%) (Table 1). Over half of the group (61\%) did not have any comorbidities. In the remaining RA patients, the most common comorbidities included arterial hypertension $(20.79 \%)$ and hypothyroidism (17.82\%). The vast majority of patients were treated with tocilizumab (67.33\%). The mean RA duration in the group studied was 13.54 \pm 9.51 years and the disease activity score was $4.8 \pm 0.8$.

Using the VAS tool, the respondents assessed the intensity of their pain at 4.4 \pm 2.7 . As for mood disorders, the mean anxiety and depression scores in the group studied were $20.0 \pm 2.3$ and $17.1 \pm 2.2$, respectively.

\section{Perception of Health and QoL}

The mean QoL perception score was 3.48 \pm 0.8 , which means that the respondents believed their QoL fell into the range between good and average (Table 1). Nearly $40 \%$ of the respondents could not clearly determine their QoL, perceiving it as neither poor nor good, and $10 \%$ believed their QoL is poor or very poor. The mean health perception score was 2.63 \pm 1 , which means that the respondents believed their health fell into the range between dissatisfactory and average. Over $50 \%$ of the respondents in total were dissatisfied and very dissatisfied with their health: $42.57 \%$ and $9.90 \%$, respectively (Table 2).

\section{QoL Assessment in Individual Domains of the WHOQOL-BREF Questionnaire}

When assessing their QoL, the respondents assigned the highest scores in the psychological domain (15.87 \pm 2.12$)$ and social relationships domain (15.45 \pm 2.09 ), and slightly lower scores in the environment domain, in which the assessment

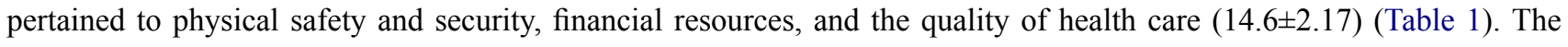
lowest scores were obtained in the physical health domain, which involves the assessment of pain and discomfort, sleep and rest, and fatigue (11.4 \pm 2.44$)$.

\section{Assessment of Anxiety and Depression Levels in the RA Patients Studied}

The analysis of anxiety with HADS demonstrated that $28.57 \%$ of respondents had "borderline" scores and $6.35 \%$ "abnormal" scores (Table 1). As for depression measured with HADS, "borderline" scores were obtained by $14.29 \%$ of respondents. The same number of patients obtained "abnormal" scores.

\section{Analysis of Correlations Between Anxiety, Depression and QoL Assessment}

The analysis of correlation between anxiety and QoL revealed that anxiety correlates significantly and negatively with QoL assessment in the psychological domain $(r=-0.472, p<0.001)$ and social domain $(r=-0.298, p=0.023)$ (Table 3). This means that the stronger the anxiety, the poorer the QoL in a given domain.

The analysis of correlation between depression and QoL showed that depression is significantly $(\mathrm{p}<0.05)$ and negatively $(\mathrm{r}<0)$ correlated with QoL in the psychological domain $(\mathrm{r}=-0.322, \mathrm{p}=0.01)$ and physical health domain $(\mathrm{r}=$

Table 2 Perception of QoL and Health in the Patients Studied

\begin{tabular}{|l|c|c|c|c|}
\hline \multirow{2}{*}{ Perception } & \multicolumn{2}{|c|}{ Perception of Quality of Life } & \multicolumn{2}{c|}{ Perception of Health } \\
\cline { 2 - 5 } & $\mathbf{n}$ & $\%$ & $\mathbf{n}$ & $\%$ \\
\hline Very poor & 2 & $1.98 \%$ & 10 & $9.90 \%$ \\
\hline Poor & 8 & $7.92 \%$ & 43 & $42.57 \%$ \\
\hline Neither poor nor good & 38 & $37.62 \%$ & 25 & $24.76 \%$ \\
\hline Good & 46 & $45.55 \%$ & 19 & $18.81 \%$ \\
\hline Very good & 7 & $6.93 \%$ & 4 & $3.96 \%$ \\
\hline
\end{tabular}


Table 3 Results of the Analysis of Correlations Between Anxiety, Depression (HADS) and QoL (WHOQOL-BREF) in RA Patients

\begin{tabular}{|l|c|c|}
\hline \multirow{2}{*}{ WHOQOL-BREF } & Anxiety & Depression \\
\cline { 2 - 3 } & \multicolumn{2}{|c|}{ Spearman Correlation Coefficient } \\
\hline Perception of quality of life & $r=-0.029, p=0.821$ & $r=-0.168, p=0.187$ \\
\hline Perception of health & $r=-0.033, p=0.796$ & $r=-0.221, p=0.05 I^{*}$ \\
\hline Physical health domain & $r=-0.097, p=0.448$ & $r=-0.209, p=0.04^{*}$ \\
\hline Psychological domain & $r=-0.472, p<0.001$ & $r=-0.322, p=0.01^{*}$ \\
\hline Social relationships domain & $r=-0.298, p=0.023$ & $r=-0.198, p=0.124$ \\
\hline Environment domain & $r=-0.055, p=0.618$ & $r=-0.104, p=0.42$ \\
\hline
\end{tabular}

Note: *Statistically significant relationship $(\mathrm{p}<0.05)$.

Abbreviation: WHOQOL-BREF, The World Health Organization Quality of Life Questionnaire.

$-0.209, \mathrm{p}=0.04$ ). In the health perception domain, the correlation is negative and on the border of significance (ie the stronger the depression, the poorer the QoL in a given domain) (Table 3).

\section{Multiple Linear Regression Analysis of Selected Variables and QoL Assessment in All Domains of the WHOQOL-BREF Questionnaire}

The analysis of selected variables and QoL domains included those variables that are important for the clinical assessment of patients with RA (Table S1).

\section{Perception of Quality of Life}

The multiple linear regression model showed that significant independent predictors of the perception of QoL are:

- Overweight: The regression parameter is -0.516 , thus overweight decreases QoL in this domain by 0.516 points on average as compared to normal weight.

- Pain: The regression parameter is -0.275 , thus each "pain point" decreases QoL in this domain by 0.275 points on average.

- Depression in HADS: The regression parameter is -0.092 , thus each "depression point" decreases QoL in this domain by 0.092 points on average.

\section{Perception of Health}

The multiple linear regression model showed that significant $(\mathrm{p}<0.05)$ independent predictors of the perception of health are:

- Depression in HADS: The regression parameter is -0.126 , thus each "depression point" decreases QoL in this domain by 0.126 points on average.

- DAS-28: The regression parameter is -0.152 , thus each point decreases QoL in this domain by 0.152 points on average.

The $\mathrm{R}^{2}$ coefficient for this model was $26.11 \%$, which means that $26.11 \%$ of the QoL variation in this domain was explained by the variables included in the model. The remaining $73.89 \%$ of the variation depends on variables not included in the model and on random factors.

\section{Physical Health Domain}

The multiple linear regression model showed that significant $(\mathrm{p}<0.05)$ independent predictors of QoL in the physical health domain are:

- Obesity: The regression parameter is -2.427 , thus obesity decreases QoL in this domain by 2.427 points on average as compared to normal weight. 
- Depression in HADS: The regression parameter is -0.244 , thus each "depression point" decreases QoL in this domain by 0.244 points on average.

- DAS-28: The regression parameter is -0.403 , thus each point decreases QoL in this domain by 0.403 points on average.

The $\mathrm{R}^{2}$ coefficient for this model was $33.15 \%$, which means that $33.15 \%$ of the QoL variation in this domain was explained by the variables included in the model. The remaining $66.85 \%$ of the variation depends on variables not included in the model and on random factors.

\section{Psychological Domain}

The multiple linear regression model showed that a significant $(\mathrm{p}<0.05)$ independent predictor of QoL in the psychological domain is:

- Disease duration: The regression parameter is -0.104 , thus each consecutive year of disease decreases QoL in this domain by 0.104 points on average.

The $\mathrm{R}^{2}$ coefficient for this model was $42.81 \%$, which means that $42.81 \%$ of the QoL variation in this domain was explained by the variables included in the model. The remaining $57.19 \%$ of the variation depends on variables not included in the model and on random factors.

\section{Social Relationships and Environment Domains}

The multiple linear regression model showed that none of the variables analysed is a significant independent predictor of QoL in the social relationships and environment domains.

The $\mathrm{R}^{2}$ coefficient for the model including the social relationships domain was $12.04 \%$, which means that $12.04 \%$ of the QoL variation in this domain was explained by the variables included in the model. The remaining $87.96 \%$ of the variation depends on variables not included in the model and on random factors.

The $\mathrm{R}^{2}$ coefficient for the model including the environment domain was $14.87 \%$, which means that $14.87 \%$ of the QoL variation in this domain was explained by the variables included in the model. The remaining $85.13 \%$ of the variation depends on variables not included in the model and on random factors.

\section{Discussion}

Rheumatoid arthritis has a major and multifaceted impact on the patient's QoL, and their physical and mental wellbeing. ${ }^{15}$ It is usually easy to identify physical disability during a clinical examination. However, clinicians may overlook social and psychological problems in RA patients. ${ }^{16}$ The results of our study demonstrated that RA patients experience decreased QoL in all the domains on the WHOQOL-BREF questionnaire, with the lowest scores observed in the physical health domain, which assesses such facets as pain, discomfort, sleep, rest, and fatigue. Over $50 \%$ of patients in our study considered their health poor or very poor, and had problems with defining their QoL, describing it as neither poor nor good. The patients assigned the lowest scores to their QoL in the physical health domain. Similar results were observed by Samar et al, with pain and role limitation being the most affected facets due to physical health. This indicates, as in the case of our study, that the physical health domain in this group of patients is more affected than the psychological domain. ${ }^{17}$

The authors of the above-cited study emphasise that RA has an impact on QoL in all its domains, and the strongest predictor is the disease activity score, DAS-28, which reduces QoL, disturbs sexuality and increases the level of depression. ${ }^{18}$ According to Da Rocha et al, there is an association between moderate to severe RA and major functional disability and morbidity. The impairment of work and activity increases together with the severity of RA. ${ }^{18}$ Samar et al observed that the majority of patients with low disease activity experienced mild to moderate anxiety. Mild anxiety was found in patients with moderate disease activity or those in remission. On the other hand, depression was observed mainly in patients with high disease activity. ${ }^{17}$

In studies published to date, the DAS-28 score has often been considered a significant and independent determinant associated with QoL and mental health. ${ }^{19,20}$ In our study, the DAS-28 score had a significant impact on the health perception and physical health domains, which corroborates findings previously published by other authors. It is worth 
noticing that patients in our study were treated with biologics, which should, as it would seem, result in better disease control and better QoL assessment. Thus, we observed an association of DAS-28 with only some of the QoL domains. The patients included in our study had high disease activity scores, which might have been caused by the previous treatment failure and the related change from first- and second-line medications to biologics. In light of the considerably high DAS-28 scores in the group studied, it is worth noting that in the case of patients treated with biologics after the previous treatment failure, the disease activity score is high at baseline. Thus, even though the patients' health improved, the outcomes were not as spectacular as the improvement seen during remission at the early stage of the disease and after effective standard treatment. It needs to be emphasised that a considerable number of studies where a large number of patients achieved remission are controlled clinical trials. Remission is a treatment outcome difficult to achieve for numerous practitioners and it most often occurs in patients with low disease activity, unlike in our study where DAS scores were high. High disease activity entails multiple problems and Wiland and other authors believe that it may be a stronger marker of deteriorated functioning than disease duration. ${ }^{21}$ Importantly, the reported data pertaining to remission in this group of patients point to low effectiveness of RA treatment, especially in Poland. ${ }^{21}$ Other studies have demonstrated that many RA patients experienced helplessness and anxiety, and that the latter strongly correlated with the DAS-28 score. ${ }^{22}$ The linear regression analysis performed in our study showed that DAS28 had a statistically significant effect on the QoL assessment, and that the higher the disease activity score, the lower the QoL.

In their study, Lempp et $\mathrm{al}^{23}$ reported an association between mental health and QoL assessed with the SF-36 questionnaire in a group of adults with early and established RA, and in a group of patients with depression. They found that the mental health and role mental domains were lowest in patients with active RA. These authors underlined that both RA and depression significantly affect mental health, and that mental symptoms are present from the earliest stages of RA and thus require an early intervention. Goma et al emphasised that depression particularly affected patients with high disease activity. ${ }^{17}$

Due to the high prevalence of mood disorders among RA patients, another objective of our study was to determine the association between depression and anxiety and QoL in this group of patients. The subject of depression and anxiety in chronic diseases is important, yet rarely discussed in the context of QoL. We did not attempt to determine the causes of low mood in our study, but the literature indicates that it may result from elevated CRP levels ${ }^{24}$ and chronic fatigue. ${ }^{25}$ Some researchers believe that depression should be regarded as a comorbidity in RA patients. ${ }^{26}$ The exact mechanisms which make depression affect QoL in RA patients are not known either. There is evidence which shows that patients with depression perceive their health condition as considerably more serious and may exaggerate their symptoms or effects of the treatment used, even if the disease and pain levels are controlled. Patients with depression and RA perceive their treatment negatively, and depression is additionally associated with impairment of their general coping capacity, especially with high pain intensity and disease exacerbation. ${ }^{27}$ As a result of these negative beliefs about the disease, depression can affect health-seeking behaviours and the use of health care, and the support received is treated as insufficient. In turn, this negative perception of the treatment process results in missed follow-up visits and a lack of consistency in taking medication. It is also worth noting that treatment of RA patients may generate higher social costs. It is less common for this group of patients to develop positive psychosocial mechanisms, which may increase illness acceptance and change the patient's attitude into a more positive one, thus improving their QoL. ${ }^{28}$ Studies by other authors have demonstrated that depression and stress may cause immune dysfunction, although there is no evidence that these factors affect disease activity, thus increasing functional disability and pain. ${ }^{28}$

Our results are in line with the findings previously published by other researchers and confirm the negative association of anxiety and depression with QoL assessment, especially in the psychological and social relationships domains for anxiety, and in the QoL perception and health perception domains for depression.

In the linear regression analysis, depression was a determinant of lowered QoL in the health and QoL perception domains. Depression and stress often accompany numerous chronic diseases. ${ }^{29}$ The patients in our study also experienced negative symptoms of mood disorders. Other authors emphasise that the prevalence of depression in patients with physical disability is even 3 times higher as compared to other individuals. ${ }^{30,31}$ In RA, the psychosomatic causes of 
depression include pain, disability and stress stemming from the fear of the future and living with a chronic illness. ${ }^{32}$ In our study, the patients described their pain as average, yet significantly limiting their daily activity.

Studies published to date have indicated that patients with arthritis and depression are less likely to engage in physical activity, are more often disabled, and experience greater joint limitation, work limitation and social activity limitation, which may play an important role in the patients' perception of their health and QoL. ${ }^{33}$

A common issue addressed by researchers is the challenge of detecting depression in RA patients. ${ }^{9}$ Depression may be difficult to diagnose in this group of patients because such somatic symptoms as fatigue, pain and insomnia can be mistakenly taken for the symptoms of the underlying disease, which delays early treatment and leads to negative health consequences. Everyday practice lacks established procedures allowing for effective diagnostics. Some tests assess only the intensity of depressive symptoms, while others detect the clinical signs of depression. ${ }^{9}$

Another problem is that to a large extent depression remains untreated in these patients. This is partly due to the fact that the physical aspects of RA attract greater focus. Another reason would be limited resources. There is a common misconception that treatment of depression is not necessary or relevant in RA patients, as the problem is only secondary to the pain and disability experienced by these patients. As a result, the problem of depression under-treatment is even more exacerbated. Currently, it is not clear how to best combine the treatment of synovitis in RA with the treatment of mental problems. The use of multidisciplinary and integrated care is the most logical solution. Management of these conditions involving multidisciplinary teams and integrated care seems to be reasonable, although there is no evidence that this approach is appropriate and will result in expected outcomes. Other studies indicate the need to use psychoeducational programmes combined with antidepressants. An important element of therapy is teaching the patient how to cope with stress and chronic illness. Studies confirm that patient education and psychoeducational programmes are highly effective in mitigating pain, depression and disability. ${ }^{34}$

To conclude, although the main aim of our study was to assess the relationship between QoL and depression, the linear regression analysis showed a statistically significant association between other clinical factors and QoL assessment. For the physical health domain this was obesity, and for the psychological domain this was disease duration (the longer the duration, the lower the QoL in this domain).

In the subject literature, the duration of chronic disease is often considered a significant determinant of QoL. However, the results published are inconsistent. Studies whose authors analyse associations between disease duration and psychosocial mechanisms determining QoL indicate that the longer the disease duration, the higher the QoL, provided that adequate mechanisms of coping with the disease are implemented. ${ }^{35}$ However, there are also studies showing that longer disease duration significantly lowers QoL. ${ }^{36-38}$ Accordingly, in the case of RA, longer disease duration may be associated with greater disheartenment with life and long-term treatment, fatigue related to frequent visits to medical facilities or increasing disability in the course of progressive inflammation, ${ }^{36}$ which in turn results in even greater mental health problems in the area of stress, depression, and discouragement. ${ }^{37,38}$

The subject of obesity as a determinant of QoL, which in our study turned out to have a negative effect in the regression analysis, is associated with the increasing problem of disability, pain, and the number of affected joints. ${ }^{38}$ There are studies which show that obesity in RA patients is associated with chronic inflammation, immune response, and chronic deficiency of vitamin D. In their study, Liu et al concluded that obesity reduces the chances of remission in RA and negatively affects disease activity and treatment outcomes reported by patients. ${ }^{39}$ It needs to be emphasised that the knowledge about the association between obesity and RA is incomplete and requires continuous research. Better understanding of interactions between arthritis and obesity will help us improve personalised treatment, targeting both the disease itself and its comorbidities and, first and foremost, enhance the patients' quality of life.

\section{Conclusions}

1. RA patients treated with biologics present a low level of health perception and an average level of QoL perception. The patients assigned the lowest scores to QoL in the physical health domain, and the highest to QoL in the psychological and social relationships domains. 
2. Depression and anxiety negatively correlate with QoL domains: the higher the anxiety level, the poorer the QoL in the psychological and social relationships domains; and the higher the depression level, the poorer the QoL in the psychological and physical health domains.

3. Independent determinants decreasing QoL are: depression, obesity, pain, and disease duration.

4. Disease activity defined by the DAS-28 scale is significantly related to the assessment of the quality of life in the health perception and physical functioning domains. The greater the disease activity, the lower the quality of life score.

\section{Study Limitation}

To our best knowledge, this is one of few studies assessing the association of depression and anxiety with QoL in patients with RA treated with biologics. However, our study had certain limitations. Firstly, the study sample was not very large. Furthermore, the study group was enrolled in one research centre and was limited to patients treated with biologics. It should be noted that in the case of patients treated with tocilizumab, this was a second- or even third-line treatment after the failure of earlier therapy. In the future, the study sample should be large and a cohort study should be conducted, comparing the outcomes in a patient group treated with different biologics and a group treated with other medications within standard care. Secondly, the patients included in the study differed with regard to disease duration and the treatment used. Data on QoL were collected at a distinct time point and thus may not have reflected the outcomes throughout the disease continuum. Another limitation of the study was the use of different criteria for the diagnosis of RA, which may influence different baseline clinical status and different response to treatment. Finally, even though the tools used in the study are standardised instruments, the lack of guidelines pertaining to QoL and depression assessment makes it impossible to compare the results obtained with the findings published in other studies, which used other standardised tools for QoL and depression assessment.

\section{Disclosure}

The authors report no conflicts of interest in this work.

\section{References}

1. Centers for Disease Control and Prevention. Arthritis in general; [updated November 13, 2014]. Available from: http://www.cdc.gov/arthritis/ basics/general.htm. Accessed November 12, 2021.

2. Alonso J, Ferrer M, Gandek B, et al. Health-related quality of life associated with chronic conditions in eight countries: results from the international quality of life assessment (IQOLA) project. Qual Life Res. 2004;13(2):283-298. doi:10.1023/B:QURE.0000018472.46236.05

3. Hootman JM, Helmick CG, Barbour KE, et al. Updated projected prevalence of self-reported doctor-diagnosed arthritis and arthritis-attributable activity limitation among US adults, 2015-2040. Arthritis Rheumatol. 2016;68:1582-1587.

4. Barbour KE, Helmick CG, Boring MA, Brady TJ. Vital signs: prevalence of doctor-diagnosed arthritis and arthritis-attributable activity limitation United States, 2013-2015. Morb Mortal Wkly Rep. 2017;66(9):246-253. doi:10.15585/mmwr.mm6609e1externalicon

5. Smolen JS, Aletaha D, Barton A, et al. Rheumatoid arthritis. Nat Rev Dis Primers. 2018;4:18001. doi:10.1038/nrdp.2018.1

6. Atkinson JH, Slater MA, Patterson TL, et al. Prevalence, onset, and risk of psychiatric disorders in men with chronic low back pain: a controlled study. Pain. 1991;45(2):111-121. doi:10.1016/0304-3959(91)90175-W

7. Gökoglu F, Özerbil T, GüvenKurultak D, et al. Depression in patients with rheumatoid arthritis: relationship between disease activity, functional status and quality of life. J Rheumatol Med Rehabil. 2008;19:23-27.

8. Mella LF, Bertolo MB, Dalgalarrondo P. Depressive symptoms in rheumatoid arthritis. Rev Bras de Psiquiatr. 2010;32(3):257-263. doi:10.1590/ S1516-44462010005000021

9. Sambamoorthi U, Shah D, Zhao X. Healthcare burden of depression in adults with arthritis. Expert Rev Pharmacoecon Outcomes Res. 2017;17 (1):53-65. doi:10.1080/14737167.2017.1281744

10. Gerhold K, Richter A, Schneider M, et al. Health-related quality of life in patients with long-standing rheumatoid arthritis in the era of biologics: data from the German biologics register RABBIT. Rheumatology. 2015;54(10):1858-1866. doi:10.1093/rheumatology/kev194

11. Scott DL, Garrood T. Quality of life measures: use and abuse. Baillieres Best Pract Res Clin Rheumatol. 2000;14(4):663-687. doi:10.1053/ berh. 2000.0106

12. Herrmann C. International experiences with the Hospital Anxiety and Depression Scale-a review of validation data and clinical results. J Psychosom Res. 1997;42(1):17-41. doi:10.1016/S0022-3999(96)00216-4

13. Downie WW, Leatham PA, Rhind VM, Pickup ME, Wright V. The visual analogue scale in the assessment of grip strength. Ann Rheum Dis. 1978;37(4):382-384. doi:10.1136/ard.37.4.382

14. R Core Team. R: a language and environment for statistical computing. Vienna, Austria: R Foundation for Statistical Computing; 2021. Available from: https://www.R-project.org/. Accessed November 10, 2021.

15. Uhlig T, Loge JH, Kristiansen IS, Kvien TK. Quantification of reduced health-related quality of life in patients with rheumatoid arthritis compared to the general population. $J$ Rheumatol. 2007;34(6):1241-1247. 
16. Tosa EE, Albu A, Popa M. The effect of physical therapy on the improvement of the quality of life in rheumatoid arthritis patients. Civil Sport. 2016;17:29-34.

17. Goma SH, Razek MRA, Nesreen M. Abdelbary. Impact of rheumatoid arthritis on the quality of life and its relation to disease activity. Egypt Rheumatol Rehabil. 2019;46(4):304-312. doi:10.4103/err.err_39_19

18. Da Rocha Castelar Pinheiro P, Khandker R, Sato R, Rose A, Piercy J. Impact of rheumatoid arthritis on quality of life, work productivity and resource utilisation: an observational, cross-sectional study in Brazil. Clin Exp Rheumatol. 2013;31(3):334-340.

19. Prevoo M, Van't Hof M, Kuper H, van Leeuwen M, van de Putte L, van Riel P. Modified disease activity scores that include twenty-eight-joint counts. Development and validation in a prospective longitudinal study of patients with rheumatoid arthritis. Arthritis Rheum. 1995;38(1):44-48. doi:10.1002/art.1780380107

20. Fransen J, Creemers M, van Riel P. Remission in rheumatoid arthritis: agreement of the disease activity score (DAS28) with the ARA preliminary remission criteria. Rheumatology. 2004;43(10):1252-1255. doi:10.1093/rheumatology/keh297

21. Matuszewska A, Wiland P. Leczenie reumatoidalnego zapalenia stawów. Prz Reumatol. 2010;2(31):7.

22. Scott DL. Radiological progression in established rheumatoid arthritis. J Rheumatol Suppl. 2004;69:55-65.

23. Lempp H, Ibrahim F, Shaw T, et al. Comparative quality of life in patients with depression and rheumatoid arthritis. Int Rev Psychiatry. 2011;23 (1):118-124. doi:10.3109/09540261.2010.545368

24. Hider SL, Tanveer W, Brownfield A, Mattey DL, Packham JC. Depression in RA patients treated with anti-TNF is common and under-recognized in the rheumatology clinic. Rheumatology. 2009;48(9):1152-1154. doi:10.1093/rheumatology/kep170

25. Wolfe F, Michaud K. Severe rheumatoid arthritis (RA), worse outcomes, comorbid illness, and sociodemographic disadvantage characterize RA patients with fibromyalgia. J Rheumatol. 2004;31(4):695-700.

26. Nichol MB, Zhang L. Depression and health-related quality of life in patients with rheumatoid arthritis. Expert Rev Pharmacoecon Outcomes Res. 2005;5(5):645-653. PMID: 19807590. doi:10.1586/14737167.5.5.645

27. Hurwicz ML, Berkanovic E. The stress process in rheumatoid arthritis. J Rheumatol. 1993;20:1836-1844.

28. Dickens C, Creed F. The burden of depression in patients with rheumatoid arthritis. Rheumatology. 2001;40(12):1327-1330. doi:10.1093/ rheumatology/40.12.1327

29. Turner J, Kelly B. Emotional dimensions of chronic disease. West J Med. 2000;172(2):124-128. doi:10.1136/ewjm.172.2.124

30. Egede LE. Failure to recognize depression in primary care: issues and challenges. J Gen Intern Med. 2007;22(5):701-703. doi:10.1007/s11606-007$0170-z$

31. Rugulies R. Depression as a predictor for coronary heart disease. a review and meta-analysis. Am J Prev Med. 2002;23(1):51-61. doi:10.1016/ s0749-3797(02)00439-7

32. Pilling S, Anderson I, Goldberg D, Meader N, Taylor C; Two Guideline Development Groups. Depression in adults, including those with a chronic physical health problem: summary of NICE guidance. BMJ. 2009;339(10):b4108. doi:10.1136/bmj.b4108

33. Joshi N, Khanna R, Shah RM. Relationship between depression and physical activity, disability, burden, and health-related quality of life among patients with arthritis. Popul Health Manag. 2015;18(2):104-114. doi:10.1089/pop.2014.0062

34. Riemsma RP, Taal E, Kirwan JR, Rasker JJ. Systematic review of rheumatoid arthritis patient education. Arthritis Rheum. $2004 ; 51(6): 1045-1059$. PMID: 15593105. doi:10.1002/art.20823

35. Iseselo MK, Kajula L, Yahya-Malima KI. The psychosocial problems of families caring for relatives with mental illnesses and their coping strategies: a qualitative urban based study in Dar Es Salaam, Tanzania. BMC Psychiatry. 2016;16(1):146. doi:10.1186/s12888-016-0857-y

36. World Health Organization. Disability and health. Available from: https://www.who.int/news-room/fact-sheets/detail/disability-and-health. Accessed November 29, 2021.

37. Pezzato S, Bonetto C, Caimmi C, et al. Depression is associated with increased disease activity and higher disability in a large Italian cohort of patients with rheumatoid arthritis. Adv Rheumatol. 2021;61(1):57. doi:10.1186/s42358-021-00214-3

38. Alvarez-Nemegyei J, Pacheco-Pantoja E, González-Salazar M, et al. Association between overweight/obesity and clinical activity in rheumatoid arthritis. Reumatol Clin. 2020;16(6):462-467. English, Spanish. doi:10.1016/j.reuma.2018.11.005

39. Liu Y, Hazlewood GS, Kaplan GG, Eksteen B, Barnabe C. Impact of obesity on remission and disease activity in rheumatoid arthritis: a systematic review and meta-analysis. Arthritis Care Res. 2017;69(2):157-165. doi:10.1002/acr.22932

Psychology Research and Behavior Management

Dovepress

Publish your work in this journal

Psychology Research and Behavior Management is an international, peer-reviewed, open access journal focusing on the science of psychology and its application in behavior management to develop improved outcomes in the clinical, educational, sports and business arenas. Specific topics covered in the journal include: Neuroscience, memory and decision making; Behavior modification and management; Clinical applications; Business and sports performance management; Social and developmental studies; Animal studies. The manuscript management system is completely online and includes a very quick and fair peer-review system, which is all easy to use. Visit http://www.dovepress.com/testimonials.php to read real quotes from published authors.

Submit your manuscript here: https:/www.dovepress.com/psychology-research-and-behavior-management-journal 